Supporting Information for:

\title{
Vertical and Directional Insertion of Helical Peptide into Lipid Bilayer Membrane
}

Koji Nakatani, Tomoyuki Morita, and Shunsaku Kimura*

Department of Material Chemistry, Graduate School of Engineering, Kyoto University,

Kyoto-Daigaku-Katsura, Nishikyo-ku, Kyoto 615-8510, Japan.

Tel: +81-75-383-2400 Fax: +81-75-383-2401 E-mail: shun@scl.kyoto-u.ac.jp

\section{The details in the synthesis}

K16, PK8, PK16, FITC-PEG, and ECz-PEG-ECz were synthesized according to Scheme S-1 by the conventional liquid-phase method. The starting octapeptide, Boc-(Lys(Z)-Aib) $)_{4}-\mathrm{OCH}_{3}$, was synthesized similarly to the method reported in the literature. ${ }^{1}$ All the intermediates were identified by ${ }^{1} \mathrm{H}$ NMR spectroscopy and some of them as well as the final products were further confirmed by FAB or MALDITOF mass spectrometry. The purity of each compound was checked by thin-layer chromatography. The solvent systems for the TLC analysis are (A) chloroform/methanol/acetic acid (95/5/3 v/v), (B) chloroform/methanol/pyridine $\quad(95 / 5 / 3 \quad \mathrm{v} / \mathrm{v}), \quad$ (C) chloroform/methanol $\quad(10 / 1 \quad \mathrm{v} / \mathrm{v}), \quad$ (D) chloroform/methanol (5/1 v/v), and (E) chloroform/methanol/ammonia water (13/5/1 v/v/v). The details in the synthesis are described below.

Boc- $\mathrm{NH}-\left(\mathrm{CH}_{2}\right)_{2}-\mathrm{CO}-\mathrm{NH}-\mathrm{ECz} . \quad$ Boc- $\beta$-alanine $(1.8 \mathrm{~g})$ and 3-amino- $\mathrm{N}$-ethylcarbazole $(2.0 \mathrm{~g})$ were dissolved in DMF, $N, N^{\prime}$-dicyclohexylcarbodiimide (DCC) $(2.4 \mathrm{~g})$ and 1-hydroxybenzotriazole $(\mathrm{HOBt})$ $(1.9 \mathrm{~g})$ were added to the solution, and the solution was stirred for at $0{ }^{\circ} \mathrm{C}$ for $30 \mathrm{~min}$ and thereafter at room temperature for $12 \mathrm{~h}$ under $\mathrm{N}_{2}$ atmosphere. After reaction, ethyl acetate was added to the solution, the solution was stirred for $30 \mathrm{~min}$ at $0{ }^{\circ} \mathrm{C}$, and the precipitated dicyclohexylurea was removed by filtration. The supernatant solution was then washed with water once, a $\mathrm{NaHCO}_{3}$ aqueous solution twice, a $\mathrm{KHSO}_{4}$ solution twice, a saturated $\mathrm{NaCl}$ solution once, in this order. The organic layer was then dried over anhydrous $\mathrm{MgSO}_{4}$, the solvent was evaporated, and the residue was purified by a silica gel column with chloroform/methanol (10/1 v/v) as eluent to afford the product (770 mg, $21 \%)$. TLC: $R_{\mathrm{f}}(\mathrm{A})=0.63, R_{\mathrm{f}}(\mathrm{B})=0.67 .{ }^{1} \mathrm{H} \mathrm{NMR}\left(400 \mathrm{MHz}, \mathrm{CDCl}_{3}\right): \delta(\mathrm{ppm}) 1.38-1.55\left(12 \mathrm{H}, \mathrm{m},\left(\mathrm{CH}_{3}\right)_{3} \mathrm{CO}\right.$, $\left.\mathrm{NCH}_{2} \mathrm{CH}_{3}\right), 2.63-2.65\left(2 \mathrm{H}, \mathrm{m}, \mathrm{NHCH}_{2} \mathrm{CH}_{2} \mathrm{CO}\right), 3.36-3.52\left(2 \mathrm{H}, \mathrm{t}, \mathrm{NHCH}_{2} \mathrm{CH}_{2} \mathrm{CO}\right), 4.13(1 \mathrm{H}, \mathrm{m}$, Lys(Z) $\left.\mathrm{C}^{\alpha} H\right), 4.31-4.36\left(2 \mathrm{H}, \mathrm{q}, \mathrm{NCH}_{2} \mathrm{CH}_{3}\right), 5.17(1 \mathrm{H}, \mathrm{t}$, urethane $\mathrm{NH}), 7.19-8.30(8 \mathrm{H}, \mathrm{m}$, carbazole $H$, amide $\mathrm{N} H$ ). 
BKZ8ECZ. Boc-(Lys(Z)-Aib) $)_{4}-\mathrm{OCH}_{3}(\mathrm{BKZ8OMe})(150 \mathrm{mg})$ was dissolved in a dioxane and methanol mixed solvent $(1 / 1 \mathrm{v} / \mathrm{v}, 3.0 \mathrm{ml})$, a $1 \mathrm{~N} \mathrm{NaOH}$ aqueous solution $(0.59 \mathrm{ml})$ was added to the solution, the solution was stirred at $50{ }^{\circ} \mathrm{C}$ for $1 \mathrm{~h}$. After reaction, the solution was neutralized by concentrated hydrochloric acid, and the solvent was evaporated. The obtained residue was dissolved in chloroform, and the solution was washed with a $\mathrm{KHSO}_{4}$ aqueous solution and saturated $\mathrm{NaCl}$ solution. The organic layer was then dried over anhydrous $\mathrm{MgSO}_{4}$, the solvent was evaporated, and the residue was washed with diisopropylether to afford Boc-(Lys(Z)-Aib) $)_{4}-\mathrm{OH}$. On the other hand, Boc-NH- $\left(\mathrm{CH}_{2}\right)_{2}-\mathrm{CO}-\mathrm{NH}-$ $\mathrm{ECz}(49 \mathrm{mg})$ was dissolved in $4 \mathrm{~N}$ hydrochloric acid/dioxane $(3.9 \mathrm{ml})$ and the solution was stirred at room temperature for $30 \mathrm{~min}$. After reaction, the solvent was evaporated, and the residue was washed with hexane to afford $\mathrm{HCl} \cdot \mathrm{NH}-\left(\mathrm{CH}_{2}\right)_{2}-\mathrm{CO}-\mathrm{NH}-\mathrm{ECz}$. Boc-(Lys(Z)-Aib $)_{4}-\mathrm{OH}(100 \mathrm{mg})$ and $\mathrm{HCl} \cdot \mathrm{NH}-$ $\left(\mathrm{CH}_{2}\right)_{2}-\mathrm{CO}-\mathrm{NH}-\mathrm{ECz} \quad(60 \mathrm{mg})$ were dissolved in DMF, O-(7-azabenzotriazol-1-yl)-1,1,3,3tetramethyluronium hexafluorophosphate (HATU) $(38 \mathrm{mg}$ ) and $N, N$-diisopropylethylamine (DIEA) (40 $\mu \mathrm{l})$ were added to the solution, and the solution was stirred at $0{ }^{\circ} \mathrm{C}$ for $3 \mathrm{~h}$ and thereafter at room temperature for $12 \mathrm{~h}$ under $\mathrm{N}_{2}$ atmosphere. After reaction, the solvent was evaporated, and the residue was purified by a Sephadex LH20 column with chloroform/methanol (1/1 v/v) as eluent to afford the product $(69 \mathrm{mg}, 59 \%)$. TLC: $R_{\mathrm{f}}(\mathrm{A})=0.70, R_{\mathrm{f}}(\mathrm{C})=0.44 .{ }^{1} \mathrm{H} \mathrm{NMR}\left(400 \mathrm{MHz}, \mathrm{CDCl}_{3}\right): \delta(\mathrm{ppm}) 1.30$ $1.80\left(60 \mathrm{H}, \mathrm{m},\left(\mathrm{CH}_{3}\right)_{3} \mathrm{CO}, \mathrm{Aib} \mathrm{CH}_{3}\right.$, Lys(Z) $\mathrm{C}^{\beta} \mathrm{H}_{2}$, Lys(Z) $\left.\mathrm{C}^{\gamma} \mathrm{H}_{2}, \mathrm{Lys}(\mathrm{Z}) \mathrm{C}^{\delta} \mathrm{H}_{2}, \mathrm{NCH}_{2} \mathrm{CH}_{3}\right), 2.63(2 \mathrm{H}, \mathrm{m}$, $\left.\mathrm{NHCH}_{2} \mathrm{CH}_{2} \mathrm{CO}\right), 2.8-3.1$ (8H, m, Lys $\left.(\mathrm{Z}) \mathrm{C}^{\varepsilon} \mathrm{H}_{2}\right), 3.46$ (1H, m, Lys $\left.(\mathrm{Z}) \mathrm{C}^{\alpha} \mathrm{H}\right), 3.52-3.74$ (4H, m, Lys $(\mathrm{Z})$ $\left.\mathrm{C}^{\alpha} \mathrm{H}, \mathrm{NHCH}_{2} \mathrm{CH}_{2} \mathrm{CO}\right), 4.13\left(1 \mathrm{H}, \mathrm{m}, \mathrm{Lys}(\mathrm{Z}) \mathrm{C}^{\alpha} \mathrm{H}\right), 4.23-4.25\left(2 \mathrm{H}, \mathrm{m}, \mathrm{NCH}_{2} \mathrm{CH}_{3}\right), 5.01-5.30(13 \mathrm{H}, \mathrm{m}$, urethane $\left.\mathrm{NH}, \mathrm{OCH}_{2} \mathrm{C}_{6} \mathrm{H}_{5}\right), 6.44-8.93\left(36 \mathrm{H}, \mathrm{m}, \mathrm{OCH}_{2} \mathrm{C}_{6} H_{5}\right.$, carbazole $H$, amide $\left.\mathrm{NH}\right)$. MS (FAB, matrix; nitrobenzylalcohol): m/z 1770.9 (calcd for $\left.\mathrm{C}_{94} \mathrm{H}_{128} \mathrm{~N}_{15} \mathrm{O}_{19}\left[(\mathrm{M}+\mathrm{H})^{+}\right] \mathrm{m} / \mathrm{z} 1771.95\right)$.

Boc-NH-(CH$)_{2}-\mathrm{NH}-\mathrm{CO}-\mathrm{PEG}-\mathrm{COOH}$. HOOC-PEG-COOH (1.0 g) was dissolved in dichloromethane, DCC (340 mg) was added to the solution, and the solution was stirred at $0{ }^{\circ} \mathrm{C}$ for $20 \mathrm{~min}$. After that, Boc-NH- $\left(\mathrm{CH}_{2}\right)_{2}-\mathrm{NH}_{2}$ was added to the solution, and the solution was stirred at room temperature for 12 h. After that, the precipitated dicyclohexylurea was removed by filtration, the solvent was evaporated, and the residue was purified by an ion-exchange column, a silica gel column with chloroform/methanol (10/1 v/v) as eluent, and a Sephadex LH20 column with methanol as eluent, in this order to afford the product. TLC: $R_{\mathrm{f}}(\mathrm{D})=0.18, R_{\mathrm{f}}(\mathrm{E})=0.54 .{ }^{1} \mathrm{H}$ NMR $\left(400 \mathrm{MHz}, \mathrm{CDCl}_{3}\right): \delta(\mathrm{ppm}) 1.33(9 \mathrm{H}, \mathrm{s}$, $\left.\left(\mathrm{CH}_{3}\right)_{3} \mathrm{CO}\right), 3.16-3.17\left(2 \mathrm{H}, \mathrm{m}, \mathrm{NHCH}_{2} \mathrm{CH}_{2} \mathrm{NH}\right), 3.29-3.30\left(2 \mathrm{H}, \mathrm{m}, \mathrm{NHCH}_{2} \mathrm{CH}_{2} \mathrm{NH}\right), 3.54-3.57(44 \mathrm{H}$, m, PEG $\left.\mathrm{CH}_{2} \mathrm{O}\left(\mathrm{CH}_{2} \mathrm{CH}_{2} \mathrm{O}\right)_{11} \mathrm{CH}_{2}\right), 3.78,3.89$ (4H, s, PEG CH $\left.\mathrm{CH}_{2} \mathrm{O}\left(\mathrm{CH}_{2} \mathrm{CH}_{2} \mathrm{O}\right)_{11} \mathrm{CH}_{2}\right)$. MS (FAB, matrix; nitrobenzylalcohol): m/z 783.4 (main peak) (calcd for $\mathrm{C}_{33} \mathrm{H}_{64} \mathrm{~N}_{2} \mathrm{NaO}_{17}\left[(\mathrm{M}+\mathrm{Na})^{+}\right] \mathrm{m} / \mathrm{z} 783.41$ ). 
Boc-PEG-KZ8ECz. BKZ8ECz (86 mg) was dissolved in trifluoroacetic acid (TFA) (860 $\mu \mathrm{l})$ with anisole $(86 \mu \mathrm{l})$, and the solution was stirred for $30 \mathrm{~min}$. After reaction, the solvent was evaporated, and the residue was washed with diisopropylether to afford TFA·HKZ8ECz. Boc-NH- $\left(\mathrm{CH}_{2}\right)_{2}-\mathrm{NH}-\mathrm{CO}-\mathrm{PEG}-$ $\mathrm{COOH}(50 \mathrm{mg})$ and TFA·HKZ8ECz (40 mg) were dissolved in DMF, HATU (26 mg) and DIEA (18 $\mu \mathrm{l})$ were added to the solution, and the solution was stirred at $0{ }^{\circ} \mathrm{C}$ for $30 \mathrm{~min}$ and thereafter at room temperature for $13 \mathrm{~h}$ under $\mathrm{N}_{2}$ atmosphere. After that, HATU (4.3 mg) and DIEA (2.0 $\left.\mu \mathrm{l}\right)$ was further added to the solution, and the solution was stirred at room temperature for $5 \mathrm{~h}$ under $\mathrm{N}_{2}$ atmosphere. After reaction, the solvent was evaporated, and the residue was purified by a Sephadex LH20 column with methanol as eluent to afford the product $(14 \mathrm{mg}, 26 \%)$. TLC: $R_{\mathrm{f}}(\mathrm{C})=0.59, R_{\mathrm{f}}(\mathrm{D})=0.34 .{ }^{1} \mathrm{H}$ NMR (400 MHz, $\left.\mathrm{CDCl}_{3}\right): \delta(\mathrm{ppm})$ 1.30-2.08 (60H, m, $\left(\mathrm{CH}_{3}\right)_{3} \mathrm{CO}, \mathrm{Aib} \mathrm{CH}_{3}, \mathrm{Lys}(\mathrm{Z}) \mathrm{C}^{\beta} \mathrm{H}_{2}, \mathrm{Lys}(\mathrm{Z}) \mathrm{C}^{\gamma} H_{2}$, Lys(Z) $\left.\mathrm{C}^{\delta} \mathrm{H}_{2}, \mathrm{NCH}_{2} \mathrm{CH}_{3}\right), 2.67$ (2H, m, $\left.\mathrm{NHCH}_{2} \mathrm{CH}_{2} \mathrm{CO}\right), 3.06-3.15\left(8 \mathrm{H}, \mathrm{m}, \mathrm{Lys}(\mathrm{Z}) \mathrm{C}^{\varepsilon} \mathrm{H}_{2}\right), 3.23$ (2H, m, $\left.\mathrm{NHCH}_{2} \mathrm{CH}_{2} \mathrm{NH}\right), \quad 3.36\left(2 \mathrm{H}, \quad \mathrm{m}, \quad \mathrm{NHCH}_{2} \mathrm{CH}_{2} \mathrm{NH}\right), \quad 3.50-3.75\left(47 \mathrm{H}, \quad \mathrm{m}, \quad \mathrm{CH}_{2} \mathrm{O}\left(\mathrm{CH}_{2} \mathrm{CH}_{2} \mathrm{O}\right)_{11} \mathrm{CH}_{2}\right.$, $\mathrm{NHCH}_{2} \mathrm{CH}_{2} \mathrm{CO}$, Lys(Z) $\left.\mathrm{C}^{\alpha} H\right), 3.80\left(2 \mathrm{H}, \mathrm{m}, \mathrm{Lys}(\mathrm{Z}) \mathrm{C}^{\alpha} \mathrm{H}\right), 3.96\left(4 \mathrm{H}, \mathrm{s}, \mathrm{CH}_{2} \mathrm{O}\left(\mathrm{CH}_{2} \mathrm{CH}_{2} \mathrm{O}\right)_{11} \mathrm{CH}_{2}\right), 4.12$ $\left(1 \mathrm{H}, \mathrm{m}, \mathrm{Lys}(\mathrm{Z}) \mathrm{C}^{\alpha} \mathrm{H}\right), 4.23-4.25\left(2 \mathrm{H}, \mathrm{m}, \mathrm{NCH}_{2} \mathrm{CH}_{3}\right), 5.01-5.30\left(13 \mathrm{H}, \mathrm{m}\right.$, urethane $\left.\mathrm{NH}, \mathrm{OCH}_{2} \mathrm{C}_{6} \mathrm{H}_{5}\right)$, 6.69-8.40 (38H, m, $\mathrm{OCH}_{2} \mathrm{C}_{6} H_{5}$, carbazole $H$, amide $\left.\mathrm{NH}\right)$. MS (FAB, matrix; nitrobenzylalcohol): m/z 2413.3 (main peak) (calcd for $\mathrm{C}_{122} \mathrm{H}_{182} \mathrm{~N}_{17} \mathrm{O}_{33}\left[(\mathrm{M}+\mathrm{H})^{+}\right] \mathrm{m} / \mathrm{z} 2414.31$ ).

PK8. Boc-PEG-KZ8ECz (14 mg) was dissolved in trifluoroacetic acid (TFA) (150 $\mu \mathrm{l})$ with anisole $(15 \mu \mathrm{l})$, and the solution was stirred for $20 \mathrm{~min}$. After reaction, the solvent was evaporated, and the residue was washed with diisopropylether to afford TFA· PEG-KZ8ECz. TFA· PEG-KZ8ECz (14 mg) was dissolved in DMF, DIEA $(1.0 \mu \mathrm{l})$ and fluorescein isothiocyanate (FITC) $(6.9 \mathrm{mg})$ were added to the solution, and the solution was stirred at room temperature for $10 \mathrm{~h}$ under $\mathrm{N}_{2}$ atmosphere. After reaction, the solvent was evaporated, and the residue was purified by a Sephadex LH20 column with methanol as eluent three times to afford the product $(11 \mathrm{mg}, 70 \%)$. TLC: $R_{\mathrm{f}}(\mathrm{D})=0.48, R_{\mathrm{f}}(\mathrm{E})=0.33$. ${ }^{1} \mathrm{H}$ NMR $(400$ $\left.\mathrm{MHz}, \mathrm{CDCl}_{3}\right): \delta(\mathrm{ppm})$ 1.30-2.08 (51H, m, Aib $\mathrm{CH}_{3}$, Lys(Z) $\mathrm{C}^{\beta} H_{2}$, Lys(Z) $\mathrm{C}^{\gamma} H_{2}$, Lys(Z) $\mathrm{C}^{\delta} H_{2}$, $\left.\mathrm{NCH}_{2} \mathrm{CH}_{3}\right), 2.63\left(2 \mathrm{H}, \mathrm{m}, \mathrm{NHCH}_{2} \mathrm{CH}_{2} \mathrm{CO}\right), 2.90-3.20\left(8 \mathrm{H}, \mathrm{m}, \mathrm{Lys}(\mathrm{Z}) \mathrm{C}^{\varepsilon} \mathrm{H}_{2}\right), 3.50-3.75(51 \mathrm{H}, \mathrm{m}$, $\mathrm{NHCH}_{2} \mathrm{CH}_{2} \mathrm{NH}, \mathrm{CH}_{2} \mathrm{O}\left(\mathrm{CH}_{2} \mathrm{CH}_{2} \mathrm{O}\right)_{11} \mathrm{CH}_{2}, \mathrm{NHCH}_{2} \mathrm{CH}_{2} \mathrm{CO}$, Lys $\left.(\mathrm{Z}) \mathrm{C}^{\alpha} H\right), 3.90\left(2 \mathrm{H}, \mathrm{m}, \operatorname{Lys}(\mathrm{Z}) \mathrm{C}^{\alpha} H\right)$, 3.96, $4.00\left(4 \mathrm{H}, \mathrm{s}, \mathrm{CH}_{2} \mathrm{O}\left(\mathrm{CH}_{2} \mathrm{CH}_{2} \mathrm{O}\right)_{11} \mathrm{CH}_{2}\right)$, 4.24-4.26 (3H, m, Lys(Z) $\left.\mathrm{C}^{\alpha} \mathrm{H}, \mathrm{NCH}_{2} \mathrm{CH}_{3}\right)$, 4.97-5.57 $\left(12 \mathrm{H}, \mathrm{m}\right.$, urethane $\left.\mathrm{N} H, \mathrm{OCH}_{2} \mathrm{C}_{6} \mathrm{H}_{5}\right), 6.60-8.84\left(49 \mathrm{H}, \mathrm{m}, \mathrm{OCH}_{2} \mathrm{C}_{6} H_{5}\right.$, carbazole $H$, FITC $H$, amide $\left.\mathrm{N} H\right)$. MS (MALDI-TOF, matrix; 2-(4-hydroxyphenylazo)benzoic acid): m/z 2724.6 (main peak) (calcd for $\left.\mathrm{C}_{138} \mathrm{H}_{184} \mathrm{~N}_{18} \mathrm{NaO}_{36} \mathrm{~S}\left[(\mathrm{M}+\mathrm{Na})^{+}\right] \mathrm{m} / \mathrm{z} 2725.28\right)$. 
$B K Z 16 E C z$ (K16). The $\mathrm{C}$ terminal of BKZ8OMe and the $\mathrm{N}$ terminal of BKZ8ECz were removed according to the procedures described above to afford BKZ8OH and TFA·HKZ8ECz. BKZ8OH $(73$ $\mathrm{mg}$ ) and TFA·HKZ8ECz (83 mg) were dissolved in DMF, HATU (37 mg) and DIEA (34 $\mu \mathrm{l})$ were added to the solution, and the solution was stirred at $0{ }^{\circ} \mathrm{C}$ for $3 \mathrm{~h}$ and thereafter at room temperature for $24 \mathrm{~h}$ under $\mathrm{N}_{2}$ atmosphere. After reaction, the solvent was evaporated, and the residue was purified by a Sephadex LH20 column with methanol as eluent to afford the product $(67 \mathrm{mg}, 43 \%)$. TLC: $R_{\mathrm{f}}(\mathrm{D})=$ 0.64, $R_{\mathrm{f}}(\mathrm{E})=0.93 .{ }^{1} \mathrm{H}$ NMR $\left(400 \mathrm{MHz} \mathrm{CDCl}_{3}\right): \delta(\mathrm{ppm}) 1.32-1.80\left(108 \mathrm{H}, \mathrm{m},\left(\mathrm{CH}_{3}\right)_{3} \mathrm{CO}, \mathrm{Aib} \mathrm{CH}_{3}\right.$, Lys(Z) $\mathrm{C}^{\beta} \mathrm{H}_{2}$, Lys(Z) $\mathrm{C}^{\gamma} \mathrm{H}_{2}$, Lys(Z) $\left.\mathrm{C}^{\delta} \mathrm{H}_{2}, \mathrm{NCH}_{2} \mathrm{CH}_{3}\right), 2.65$ (2H, m, NHCH $\left.\mathrm{CH}_{2} \mathrm{CO}\right), 2.86-3.07$ (17H, m, Lys $(\mathrm{Z}) \mathrm{C}^{\alpha} \mathrm{H}$, Lys $\left.(\mathrm{Z}) \mathrm{C}^{\varepsilon} \mathrm{H}_{2}\right), 3.62-3.77\left(8 \mathrm{H}, \mathrm{m}, \mathrm{NHCH}_{2} \mathrm{CH}_{2} \mathrm{CO}, \mathrm{Lys}(\mathrm{Z}) \mathrm{C}^{\alpha} H\right), 4.23-4.25$ (3H, m, $\mathrm{NCH}_{2} \mathrm{CH}_{3}$, Lys $\left.(\mathrm{Z}) \mathrm{C}^{\alpha} H\right), \quad 5.00-5.40\left(25 \mathrm{H}, \mathrm{m}\right.$, urethane $\left.\mathrm{NH}, \mathrm{OCH}_{2} \mathrm{C}_{6} \mathrm{H}_{5}\right), 6.68-8.93(62 \mathrm{H}, \mathrm{m}$, $\mathrm{OCH}_{2} \mathrm{C}_{6} H_{5}$, carbazole $H$, amide $\mathrm{N} H$ ). $\mathrm{MS}(\mathrm{FAB}$, matrix; nitrobenzylalcohol): m/z 3159.7 (calcd for $\left.\mathrm{C}_{166} \mathrm{H}_{228} \mathrm{~N}_{27} \mathrm{O}_{35}\left[(\mathrm{M}+\mathrm{H})^{+}\right] \mathrm{m} / \mathrm{z} 3160.69\right)$.

Boc-PEG-KZ16ECz. BKZ16ECz (80 mg) was dissolved in trifluoroacetic acid (TFA) (2.0 ml) with anisole $(200 \mu \mathrm{l})$, and the solution was stirred for $20 \mathrm{~min}$. After reaction, the solvent was evaporated, and the residue was washed with diisopropylether to afford TFA $\cdot \mathrm{HKZ16ECz}$. Boc-NH- $\left(\mathrm{CH}_{2}\right)_{2}-\mathrm{NH}-\mathrm{CO}-$ PEG-COOH (54 mg) and TFA·HKZ16ECz (80 mg) were dissolved in DMF, HATU (43 mg) and DIEA $(34 \mu \mathrm{l})$ were added to the solution, and the solution was stirred at $0{ }^{\circ} \mathrm{C}$ for $30 \mathrm{~min}$ and thereafter at room temperature for $11 \mathrm{~h}$ under $\mathrm{N}_{2}$ atmosphere. After that, HATU $(5.0 \mathrm{mg})$ and DIEA (2.3 $\left.\mu \mathrm{l}\right)$ was further added to the solution, and the solution was stirred at room temperature for $6 \mathrm{~h}$ under $\mathrm{N}_{2}$ atmosphere. After reaction, the solvent was evaporated, and the residue was purified by a Sephadex LH20 column with methanol as eluent to afford the product $(37 \mathrm{mg}, 39 \%)$. TLC: $R_{\mathrm{f}}(\mathrm{C})=0.66, R_{\mathrm{f}}(\mathrm{D})=0.36$. ${ }^{1} \mathrm{H}$ NMR (400 MHz, $\left.\mathrm{CDCl}_{3}\right): \delta(\mathrm{ppm})$ 1.18-2.11 (108H, m, $\left(\mathrm{CH}_{3}\right)_{3} \mathrm{CO}, \operatorname{Aib~} \mathrm{CH}_{3}, \mathrm{Lys}(\mathrm{Z}) \mathrm{C}^{\beta} \mathrm{H}_{2}$, Lys(Z) $\mathrm{C}^{\gamma} \mathrm{H}_{2}$, Lys(Z) $\mathrm{C}^{\delta} \mathrm{H}_{2}, \mathrm{NCH}_{2} \mathrm{CH}_{3}$ ), 2.68 (2H, m, NHCH $\left.\mathrm{CH}_{2} \mathrm{CO}\right), 2.90-3.15$ (16H, m, Lys(Z) $\left.\mathrm{C}^{\varepsilon} H_{2}\right), 3.19$ (2H, m, $\left.\mathrm{NHCH}_{2} \mathrm{CH}_{2} \mathrm{NH}\right), 3.32\left(2 \mathrm{H}, \mathrm{m}, \mathrm{NHCH}_{2} \mathrm{CH}_{2} \mathrm{NH}\right), 3.41-3.73\left(47 \mathrm{H}, \mathrm{m}, \mathrm{CH}_{2} \mathrm{O}\left(\mathrm{CH}_{2} \mathrm{CH}_{2} \mathrm{O}\right)_{11} \mathrm{CH}_{2}\right.$, $\mathrm{NHCH}_{2} \mathrm{CH}_{2} \mathrm{CO}$, Lys(Z) $\left.\mathrm{C}^{\alpha} H\right), 3.91\left(4 \mathrm{H}, \mathrm{s}, \mathrm{CH}_{2} \mathrm{O}\left(\mathrm{CH}_{2} \mathrm{CH}_{2} \mathrm{O}\right)_{11} \mathrm{CH}_{2}\right), 4.10\left(1 \mathrm{H}, \mathrm{m}, \mathrm{Lys}(\mathrm{Z}) \mathrm{C}^{\alpha} H\right), 4.17-$ $4.19\left(2 \mathrm{H}, \mathrm{m}, \mathrm{NCH}_{2} \mathrm{CH}_{3}\right), 4.94-5.35\left(25 \mathrm{H}, \mathrm{m}\right.$, urethane $\left.\mathrm{NH}, \mathrm{OCH}_{2} \mathrm{C}_{6} \mathrm{H}_{5}\right), 6.65-8.34(66 \mathrm{H}, \mathrm{m}$, $\mathrm{OCH}_{2} \mathrm{C}_{6} \mathrm{H}_{5}$, carbazole $H$, amide $\mathrm{NH}$ ). MS (MALDI-TOF, matrix; 2-(4-hydroxyphenylazo)benzoic acid): $\mathrm{m} / \mathrm{z} 3824.6$ (main peak) (calcd for $\mathrm{C}_{194} \mathrm{H}_{281} \mathrm{~N}_{29} \mathrm{NaO}_{49}\left[(\mathrm{M}+\mathrm{Na})^{+}\right] \mathrm{m} / \mathrm{z} 3826.04$ ).

PK16. Boc-PEG-KZ16ECz (15 mg) was dissolved in trifluoroacetic acid (TFA) $(150 \mu \mathrm{l})$ with anisole $(15 \mu \mathrm{l})$, and the solution was stirred for $20 \mathrm{~min}$. After reaction, the solvent was evaporated, and the residue was washed with diisopropylether to afford TFA· PEG-KZ16ECz. TFA· PEG-KZ16ECz (15 
$\mathrm{mg}$ ) was dissolved in DMF, DIEA $(1.0 \mu \mathrm{l})$ and fluorescein isothiocyanate (FITC) $(7.5 \mathrm{mg})$ were added to the solution, and the solution was stirred at room temperature for $10 \mathrm{~h}$ under $\mathrm{N}_{2}$ atmosphere. After reaction, the solvent was evaporated, and the residue was purified by a Sephadex LH20 column with methanol as eluent three times to afford the product $(5.2 \mathrm{mg}, 32 \%)$. TLC: $R_{\mathrm{f}}(\mathrm{D})=0.47, R_{\mathrm{f}}(\mathrm{E})=0.34$. ${ }^{1} \mathrm{H}$ NMR (400 MHz, $\left.\mathrm{CDCl}_{3}\right): \delta(\mathrm{ppm})$ 1.30-2.08 (99H, m, Aib $\mathrm{CH}_{3}$, Lys(Z) $\mathrm{C}^{\beta} H_{2}, \operatorname{Lys}(\mathrm{Z}) \mathrm{C}^{\gamma} H_{2}$, Lys $(\mathrm{Z})$ $\left.\mathrm{C}^{\delta} \mathrm{H}_{2}, \mathrm{NCH}_{2} \mathrm{CH}_{3}\right), 2.74\left(2 \mathrm{H}, \mathrm{m}, \mathrm{NHCH}_{2} \mathrm{CH}_{2} \mathrm{CO}\right), 2.80-3.20\left(16 \mathrm{H}, \mathrm{m}, \mathrm{Lys}(\mathrm{Z}) \mathrm{C}^{\varepsilon} H_{2}\right), 3.42-3.61(51 \mathrm{H}, \mathrm{m}$, $\mathrm{NHCH}_{2} \mathrm{CH}_{2} \mathrm{NH}, \mathrm{CH}_{2} \mathrm{O}\left(\mathrm{CH}_{2} \mathrm{CH}_{2} \mathrm{O}\right)_{11} \mathrm{CH}_{2}, \mathrm{NHCH}_{2} \mathrm{CH}_{2} \mathrm{CO}$, Lys(Z) $\left.\mathrm{C}^{\alpha} H\right), 3.71\left(2 \mathrm{H}, \mathrm{m}, \operatorname{Lys}(\mathrm{Z}) \mathrm{C}^{\alpha} H\right)$, $3.91\left(4 \mathrm{H}, \mathrm{s}, \mathrm{CH}_{2} \mathrm{O}\left(\mathrm{CH}_{2} \mathrm{CH}_{2} \mathrm{O}\right){ }_{11} \mathrm{CH}_{2}\right), 4.18-4.20$ (3H, m, Lys(Z) $\left.\mathrm{C}^{\alpha} \mathrm{H}, \mathrm{NCH}_{2} \mathrm{CH}_{3}\right), 4.89-5.54$ (24H, m, urethane $\left.\mathrm{N} H, \mathrm{OCH}_{2} \mathrm{C}_{6} \mathrm{H}_{5}\right), 6.60-8.35\left(77 \mathrm{H}, \mathrm{m}, \mathrm{OCH}_{2} \mathrm{C}_{6} H_{5}\right.$, carbazole $H$, FITC $H$, amide $\left.\mathrm{N} H\right)$. MS (MALDI-TOF, matrix; 2-(4-hydroxyphenylazo)benzoic acid): m/z 4113.6 (main peak) (calcd for $\left.\mathrm{C}_{210} \mathrm{H}_{284} \mathrm{~N}_{30} \mathrm{NaO}_{52} \mathrm{~S}\left[(\mathrm{M}+\mathrm{Na})^{+}\right] \mathrm{m} / \mathrm{z} 4115.02\right)$.

FITC-PEG. FITC (18 mg) and 11-amino-3,6,9-trioxaundecanol (18 mg) were dissolved in DMF, and the solution was stirred at room temperature for $12 \mathrm{~h}$ under $\mathrm{N}_{2}$ atmosphere. After reaction, the solvent was evaporated, and the residue was purified by a Sephadex LH20 column with methanol as eluent three times to afford the product. TLC: $R_{\mathrm{f}}(\mathrm{D})=0.29, R_{\mathrm{f}}(\mathrm{E})=0.35 .{ }^{1} \mathrm{H}$ NMR $\left(400 \mathrm{MHz}, \mathrm{CD}_{3} \mathrm{OD}\right): \delta(\mathrm{ppm})$ 3.53-3.72 (16H, m, $\left.\left(\mathrm{CH}_{2} \mathrm{CH}_{2} \mathrm{O}\right)_{4}-\mathrm{H}\right), \quad 6.45-7.87 \quad(9 \mathrm{H}, \quad \mathrm{m}, \quad$ FITC $H)$. MS (FAB, matrix; nitrobenzylalcohol): m/z 583.2 (calcd for $\mathrm{C}_{29} \mathrm{H}_{31} \mathrm{~N}_{2} \mathrm{O}_{9} \mathrm{~S}\left[(\mathrm{M}+\mathrm{H})^{+}\right] \mathrm{m} / \mathrm{z} 583.18$ ).

$E C z-P E G-E C z$. HOOC-PEG-COOH (53 mg) and 3-amino- $N$-ethylcarbazole (190 mg) were dissolved in DMF, HATU (50 mg) and DIEA (69 $\mu \mathrm{l})(340 \mathrm{mg})$ was added to the solution, and the solution was stirred at $0{ }^{\circ} \mathrm{C}$ for $30 \mathrm{~min}$ and thereafter at room temperature for $11 \mathrm{~h}$. After reaction, the solvent was evaporated, and the residue was purified by a Sephadex LH20 column with methanol as eluent to afford the product. $\quad$ TLC: $R_{\mathrm{f}}(\mathrm{C})=0.44, R_{\mathrm{f}}(\mathrm{E})=0.91 .{ }^{1} \mathrm{H} \mathrm{NMR}\left(400 \mathrm{MHz}, \mathrm{CDCl}_{3}\right): \delta(\mathrm{ppm}) 1.42(6 \mathrm{H}, \mathrm{t}$, $\left.\mathrm{NCH}_{2} \mathrm{CH}_{3}\right), 2.63-2.65\left(2 \mathrm{H}, \mathrm{m}, \mathrm{NHCH}_{2} \mathrm{CH}_{2} \mathrm{CO}\right), 3.41-3.85\left(40 \mathrm{H}, \mathrm{m}, \mathrm{CH}_{2} \mathrm{O}\left(\mathrm{CH}_{2} \mathrm{CH} \mathrm{H}_{2} \mathrm{O}\right)_{11} \mathrm{CH}_{2}\right), 4.16$, $4.30\left(4 \mathrm{H}, \mathrm{s}, \mathrm{CH}_{2} \mathrm{O}\left(\mathrm{CH}_{2} \mathrm{CH}_{2} \mathrm{O}\right){ }_{11} \mathrm{CH}_{2}\right), 4.34\left(4 \mathrm{H}, \mathrm{q}, \mathrm{NCH}_{2} \mathrm{CH}_{3}\right), 7.18-8.87$ (16H, m, carbazole $H$, amide $\mathrm{NH}$ ). MS (FAB, matrix; nitrobenzylalcohol): m/z 1003.5 (main peak) (calcd for $\mathrm{C}_{54} \mathrm{H}_{75} \mathrm{~N}_{4} \mathrm{O}_{14}\left[(\mathrm{M}+\mathrm{H})^{+}\right]$ $\mathrm{m} / \mathrm{z}$ 1003.53). 
Scheme S-1. Synthetic schemes of K16, PK8, PK16, FITC-PEG, and ECz-PEG-ECz.
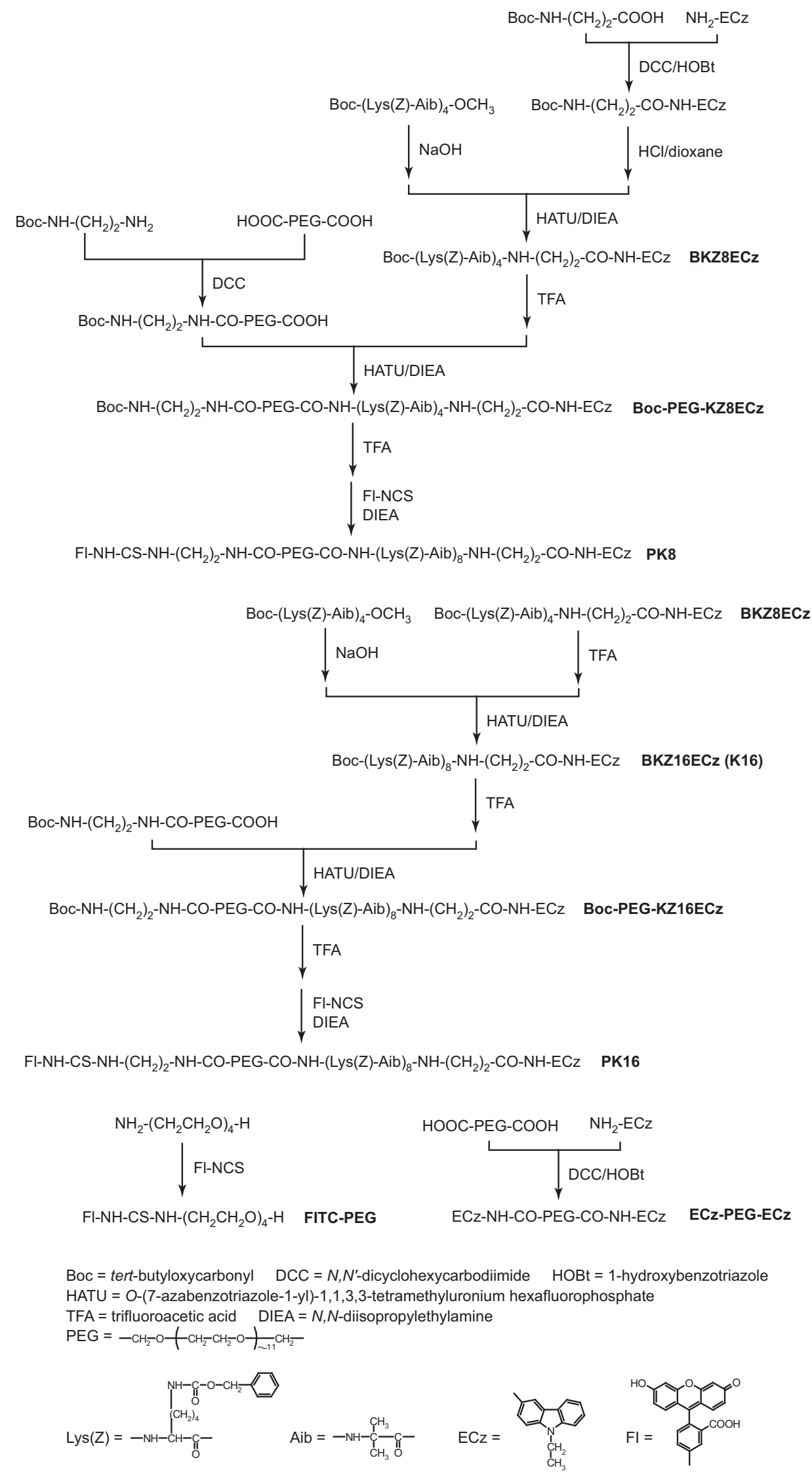